Bretonya Phillips, Maria L.

\title{
Severe Non-Opioid-Induced Pruritus Following Spinal Block
}

\section{Felicia Davis-Fourte,} Bozana Alexander

John. H. Stroger, Jr. Hospital of Cook County, Chicago, IL 60612, USA

\section{Correspodence}

Bozana Alexander, MD

John H. Stroger, Jr. Hospital of Cook County, Chicago, Illinois 60612, USA

\begin{abstract}
This case report describes an occurrence of intractable localized pruritus after spinal anesthesia in the absence of neuraxial opioid. Pruritus was confined to soles of booth feet. Symptoms were attributed to possible subclinical diabetic distal sensory neuropathy. Pruritus was refractory to IV diphenhydramine, IV lidocaine but responded quickly to subhypnotic dose of IV propofol. The close relationship between pathways for pain and pruritus may result in severe and unusual symptoms. Diagnostic workup should include tests for distal sensory neuropathy. Further understanding of neuropathic pruritus is needed.
\end{abstract}

\section{KEY WORDS}

Spinal block, non-opioid-induced, propofol.

A 57-year-old, $122 \mathrm{~kg}$, 6'1" male, had a past medical history of hypertension, glucose intolerance, benign prostatic hypertrophy and right-sided Bell's palsy. This patient was admitted for cystolithotrypexy under spinal anesthesia. Subarrachnoid block produced a prompt onset of anesthesia with satisfactory sensory blockade to the level of the tenth thoracic dermatome. Propofol infusion was maintained for sedation, and a Ramsay scale of 5 was obtained. The patient tolerated the procedure with no untoward events and was transferred to the Post Anesthesia Care unit. Approximately 60 minutes after arrival in the recovery room, the patient complained of intense itching confined to soles of both feet. He reported the onset of this discomfort soon after he awoke in the OR. Upon evaluation, the patient had no motor block and sensory recovery to the level of L3 dermatome. Treatment of the present severe pruritis was initiated with intravenous lidocaine. Ten minutes later the patient reported no relief (verbal analog scales 10/10). His discomfort was so great that physical restraint was required to keep him from getting of the bed and excoriating his feet. Subsequently, IV diphenhydramine controlled his agitation but had no effect on the severe itching. Subhypnotic propofol infusion was started with a total infusion time of thirty minutes. The itching subsided dramatically after five minutes and after 20 min was no longer present. At that time, there were a complete resolution of sensory and motor block and the patient was discharged from the recovery room and advised to pursue a work up for diabetic neuropathy and to seek follow up in the pain clinic if symptoms recurred. In the mean time patient had another cystoscopy procedure under spinal anesthesia and had the exactly the same course in the Post Anesthesia Care unit. The only difference was that prior to the placement of subarrachnoid block he received IV fentanyl as a premedication.

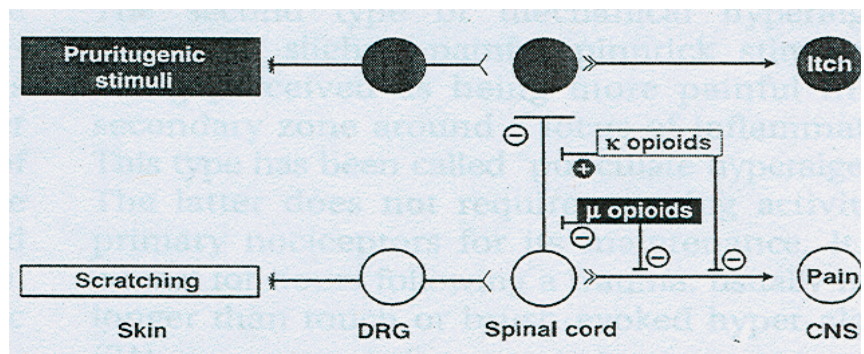

FIG. 1. Simplified schematic view of central interaction between pain and itch under physiological conditions. While having a similar inhibitory effect on the pain processing, $\mu$ and kappa opioids differentially modify the spinal itch processing. DRG: dorsal root ganglion; CNS: central nervous system.

\section{Discussion}

The close relationship between pathways for pain and pruritus may result in severe and unusual symptoms..$^{1-3}$ We report an episode of neuropathic symptoms following neuraxial anesthesia during the period prior to restoration of full sensation. The severe pruritus in the absence of 
neuraxial opioid during spinal anesthesia could be an early manifestation of latent systemic disease, such as diabetic neuropathy.

Although the itch sensation seems to be transmitted via the C-fibers, which are different from those that transmit pain, increasing evidence supports an interrelation between these two distinct sensations. Painful stimuli, such as thermal, mechanical, or chemical, can inhibit itching, and inhibition of pain processing may enhance itching. Opioid receptors appear to be involved in the interactions between itching and pain (Figure 1). ${ }^{1}$ Mild paresthesias during the return of full sensation are common. Possibly, our patient perceived paresthesia as an extremely irritating pruritus due to the presence of subclinical neuropathy.

\section{References}

1. Igic R, Alexander B. Neurophysiologic approach to pruritis and pain in cancer patient. J BUON, 2006;11:143-52.

2. Schmelz M. Itch and pain. Dermatol Ther 2005;18:304-7. 